\title{
The Cardinal Spline Methods for the Numerical Solution of Nonlinear Integral Equations
}

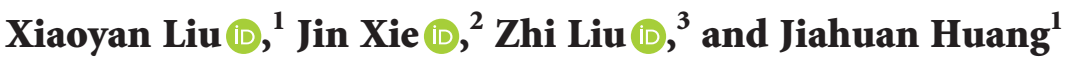 \\ ${ }^{1}$ Department of Mathematic, University of La Verne, La Verne, CA 91750, USA \\ ${ }^{2}$ School of Artificial Intelligence and Big Data, Hefei University, Hefei 230601, China \\ ${ }^{3}$ School of Mathematic, Hefei University of Technology, Hefei 230001, China \\ Correspondence should be addressed to Jin Xie; xiejin@hfuu.edu.cn
}

Received 20 October 2019; Revised 27 December 2019; Accepted 6 January 2020; Published 12 February 2020

Guest Editor: Shaohui Wang

Copyright (c) 2020 Xiaoyan Liu et al. This is an open access article distributed under the Creative Commons Attribution License, which permits unrestricted use, distribution, and reproduction in any medium, provided the original work is properly cited.

\begin{abstract}
In this study, an effective technique is presented for solving nonlinear Volterra integral equations. The method is based on application of cardinal spline functions on small compact supports. The integral equation is reduced to a system of algebra equations. Since the matrix for the system is triangular, it is relatively straightforward to solve for the unknowns and an approximation of the original solution with high accuracy is accomplished. Several cardinal splines are employed in the paper to enhance the accuracy. The sufficient condition for the existence of the inverse matrix is examined, and the convergence rate is analyzed. We compare our method with other methods proposed in recent papers and demonstrated the advantage of our method with several examples.
\end{abstract}

\section{Introduction}

Integral equations appear in many fields, including dynamic systems, mathematical applications in economics, communication theory, optimization and optimal control systems, biology and population growth, continuum and quantum mechanics, kinetic theory of gases, electricity and magnetism, potential theory, geophysics, and theoreticalmodel chemistry. Many differential equations with boundary values can be reformulated as integral equations. There are also some problems that can be expressed only in terms of integral equations. Many differential equations with boundary values can be reformulated as integral equations; for example, for chemical integral equations with boundaries, Polyanin summarized different solutions of integral equations in [1-3], published in 2013 and 2016. In [4-6], we discussed numerical methods using cardinal splines in solving systems of linear integral equations. In this paper, we are going to explore the applications of cardinal splines in solving nonlinear integral equations.
We are interested in the Volterra integral equations of the second kind:

$$
y(x)=g(x)+\int_{a}^{x} K(x, t, y(t)) \mathrm{d} t, \quad x \in(a, b),
$$

where the kernel $K(x, t, y)$ and $g(x)$ are known functions and $y(x)$ is to be determined.

This paper is divided into six sections. In Sections 2 and 3 , two univariate cardinal continuous splines on small compact supports are constructed and properties are given. In Section 4, the applications of cardinal splines on solving integral equations are explored. The unknown function is expressed as a linear combination of horizontal translations of a cardinal spline function. Then, a system of equations on the coefficients are deducted. We can solve the system, and a good approximation of the original solution is obtained. The sufficient condition for the existence of the inverse matrix is discussed, and the convergence is investigated. In Section 5, the numerical examples are given. The nonlinear system on unknowns is solved, and an accurate approximation of the 
original solution is obtained in each case. Section 6 contains the conclusion remarks.

\section{Cardinal Splines with Small Compact Supports}

Since the paper [7] by Schoenberg published in 1946, spline functions have been studied by many scholars. Spline functions have excellent properties, and applications are endless (for examples, cf [2]). The spline functions on uniform partitions are simple to construct and easy to apply and are sufficient for a variety of applications.

The starting point is frequently the zero degree polynomial $B$-spline (see Figure 1), with the integral iteration formula

$$
\begin{aligned}
& B_{0}(x)= \begin{cases}1, & -\frac{1}{2}<x<\frac{1}{2}, \\
0, & \text { elsewhere, }\end{cases} \\
& B_{n}(x)=\int_{-(1 / 2)}^{1 / 2} B_{n-1}(x+t) \mathrm{d} t, \quad n=1,2,3, \ldots
\end{aligned}
$$

We could construct higher-order polynomial spline functions with a higher degree of smoothness. More specifically, $B_{1}(x)$ has the expression (see Figure 2 ) as follows:

$$
B_{1}(x)=\frac{1}{2}|x-1|+\frac{1}{2}|x+1|-|x| .
$$

$B_{n}(x)$ are called one-dimensional $B$-splines, which are polynomial splines and have small supports $\quad(-((n+1) / 2),(n+1) / 2)$, i.e., $\quad B_{n}(x)=0$ for $x>(n+1) / 2$ or $<-((n+1) / 2)$ and excellent traits (cf [8]). In my previous papers $[4,5]$, low-degree orthonormal spline and cardinal spline functions with small compact supports were applied in solving the second kind of Volterra integral equations. In this paper, we use the notation $\|u\|_{A}=\max _{x \in A}|u(x)|$.

Let $B_{1, h}(x)=B_{1}(x / h)$. It is proved that

$$
\sum_{m=-\infty}^{\infty}(a+b m h) B_{1, h}(x-m h)=(a+b x) .
$$

Note that this particular $B$-spline is also a cardinal spline; therefore, it is straightforward to apply it in interpolations. As far as the convergence rate of interpolation is concerned, we have the following proposition (cf [9-12]).

Proposition 1. Given that $f(x) \in C[a, b], f^{\prime}(x)$ exists and is bounded in $[a, b]$. Let $n$ be an integer; $h=(b-a) / n$; let $x_{i}=a+i h ; f_{i}=f\left(x_{i}\right), i=0,1,2, \ldots, n$, as follows:

$$
\Omega f(x)=\sum_{k=0}^{n} f_{k} B_{1, h}(x-k h) \text {. }
$$

Then,

$$
\|\Omega f(x)-f(x)\|_{[a, b]} \leq 3\left\|f^{\prime}(x)\right\|_{[a, b]} h^{2}
$$

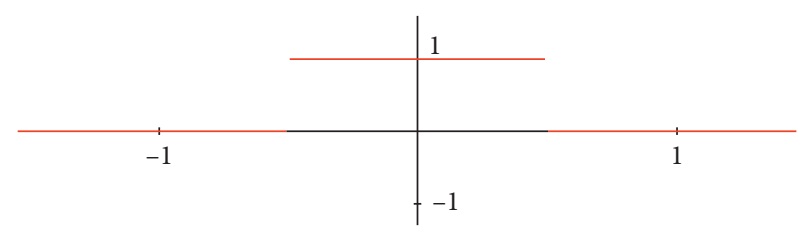

Figure 1: The graph of $B_{0}(x)$.

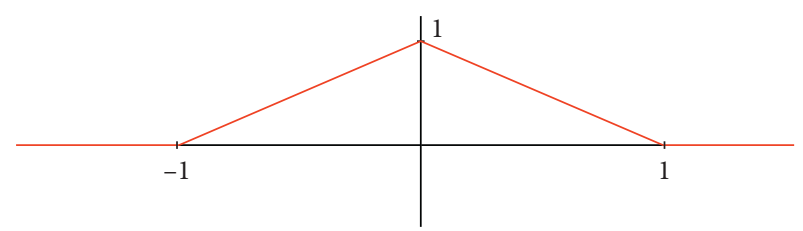

FIGURE 2: The graph of $B_{1}(x)$.

where $\Omega$ denotes the approximation polynomial of $f(x)$.

If $f(x) \in C(-\infty, \infty)$ and $f^{\prime}(x)$ exists and is bounded, let $h$ be a real number, and let $f_{i}=f(i h), i=0,1,2, \ldots$ :

$$
\Omega f(x)=\sum_{k=-\infty}^{\infty} f_{k} B_{1, h}(x-k h) .
$$

Then,

$$
\|\Omega f(x)-f(x)\|_{(-\infty, \infty)} \leq 3\left\|f^{\prime}(x)\right\|_{(-\infty, \infty)} h^{2} .
$$

\section{A Univariate $C^{2}$ Cardinal Spline}

By cardinal conditions (cf [13]), we mean that let $L(x)$ be a function, $\left\{x_{i}\right\}, i=0, \pm 1, \pm 2, \pm 3, \ldots$ be interpolation points; then,

$$
L\left(x_{i}\right)=\left\{\begin{array}{ll}
1, & i=0, \\
0, & i \neq 0,
\end{array} \quad i=0, \pm 1, \pm 2, \pm 3, \pm 4, \ldots\right.
$$

The cardinal spline that was originally given in [10] is based on $B_{3}(x)$ using the similar process as in Section 2. Let

$$
\begin{aligned}
L_{3}(x) & =6 B_{3}(x)-60 \int_{-(1 / 2)}^{1 / 2} t^{2} B_{2}(x+t) \mathrm{d} t \\
& =\int_{-(1 / 2)}^{1 / 2}\left(6-60 t^{2}\right) B_{2}(x+t) \mathrm{d} t
\end{aligned}
$$

Figure 3 shows the graph of $L_{3}(x)$.

Then, $L_{3}(x / h)$ satisfies the above cardinal condition when $x_{i}=i h, i=0, \pm 1, \pm 2, \pm 3, \ldots$. Notice that by the construction $\quad L_{3}(x) \in C^{2}(-\infty, \infty) . \quad L_{3}(x)=0$ for $x \in(-\infty,-2) \cup(2, \infty) . L_{3}(x)$ is a polynomial of degree $\leq 5$ in each subinterval $(-2,-1),(-1,0),(0,1)$, and $(1,2)$ of its support.

Furthermore, from direct calculation, we deduct the following two propositions (cf [10]). 


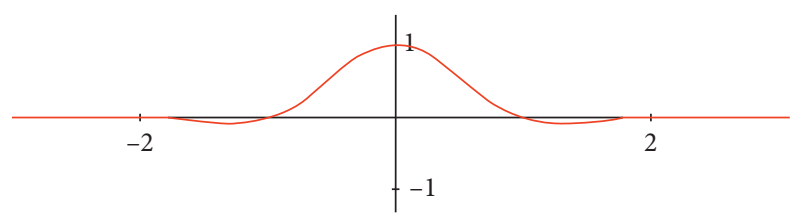

Figure 3: The graph of $L_{3}(x)$.

Proposition 2. Let $L_{3}(x)$ be the cardinal spline constructed above; then,

$$
\begin{gathered}
\sum_{m=-\infty}^{\infty} L_{3}\left(\frac{x}{h}-m\right)\left(\alpha(i h)^{2}+\beta(i h)+\gamma\right)=\alpha x^{2}+\beta x+\gamma, \\
\left\|\sum_{m=-\infty}^{\infty} L_{3}\left(\frac{x}{h}-m\right)(i h)^{3}-x^{3}\right\| \leq \frac{1}{6} h^{3},
\end{gathered}
$$

where $\alpha, \beta$, and $\gamma$ are any complex number.

Proposition 3. If $f(x) \in C^{3}[a, b]$, let $h=(b-a) / n, n$ be an integer; let $f_{i}=f(a+i h), i=0,1,2, \ldots$ :

$$
\begin{aligned}
\Omega f(x)= & \left(3 f_{0}-3 f_{1}+f_{2}\right) L_{3}\left(\frac{x}{h}+1\right)+\sum_{k=0}^{n} f L_{3}\left(\frac{x}{h}-k\right)_{i} \\
& +\left(3 f_{n}-3 f_{n-1}+f_{n-2}\right) L_{3}\left(\frac{x}{h}-n-1\right) .
\end{aligned}
$$

Then,

$$
\|\Omega f(x)-f(x)\|_{[a, b]} \leq 7\left\|f^{\prime \prime \prime}(x)\right\|_{[a, b]} h^{3} .
$$

If $f(x) \in C^{3}(-\infty, \infty)$ and is bounded, let $h$ be a real number; let $f_{i}=f(i h), i=0,1,2, \ldots$ :

$$
\Omega f(x)=\sum_{k=-\infty}^{\infty} f_{k} L_{3}\left(\frac{x}{h}-k\right) .
$$

Then,

$$
\|\Omega f(x)-f(x)\|_{(-\infty, \infty)} \leq 7\left\|f^{\prime \prime \prime}(x)\right\|_{(-\infty, \infty)} h^{3} .
$$

\section{Numerical Methods Solving Integral Equations}

4.1. Method 1-V for Solving the Volterra Integral Equation. As for the Volterra integral equations (1), we solve it in an interval $[a, b]$. Again, we let $h=(b-a) / n$ and $x_{i}=a+i h, i=0,1, \ldots, n$. Furthermore, plugging $f(x)=$ $\sum_{k=0}^{n} c_{k} B_{1, h}\left(x-x_{k}\right), K(x, t, y(t))=\sum_{i=0}^{n} \sum_{j=0}^{n} K\left(x_{i}, x_{j}, c_{j}\right)$

$B_{1, h}\left(x-x_{i}\right) B_{1, h}\left(t-x_{j}\right)$, and $g(x)=\sum_{k=0}^{n} g\left(x_{k}\right) B_{1, h}(x-$ $\left.x_{k}\right)$, we get

$$
\begin{aligned}
& \sum_{k=0}^{n} c_{k} B_{1, h}\left(x-x_{k}\right)-\sum_{i=0}^{n} \sum_{j=0}^{n} B_{1, h}\left(x-x_{i}\right) \int_{a}^{x} K\left(x_{i}, x_{j}, c_{j}\right) \\
& \quad \cdot B_{1, h}\left(t-x_{j}\right) \mathrm{d} t \\
& =\sum_{k=0}^{n} g\left(x_{k}\right) B_{1, h}\left(x-x_{k}\right) .
\end{aligned}
$$

Let $x=x_{s}$; we arrive at

$$
\begin{aligned}
& c_{s}-\sum_{j=0}^{n} K\left(x_{s}, x_{j}, c_{j}\right) \int_{a}^{x_{s}} B_{1, h}\left(t-x_{j}\right) \mathrm{d} t \\
& =g\left(x_{s}\right), \quad s=0,1,2,3,4, \ldots, n,
\end{aligned}
$$

which is a simple system of $n+1$ nonlinear equations of unknowns $\left\{c_{0}, c_{1}, \ldots, c_{n}\right\}$. Notice that this is a triangular system and it is solvable (the solution may not be unique because it is not linear):

$$
\begin{aligned}
c_{0} & =g\left(x_{0}\right), \\
c_{1} & -\frac{h}{2} \sum_{j=0}^{1} K\left(x_{1}, x_{j}, c_{j}\right)=g\left(x_{1}\right), \\
c_{s}- & \frac{h}{2}\left(K\left(x_{s}, x_{0}, c_{0}\right)+2 \sum_{j=1}^{s-1} K\left(x_{s}, x_{j}, c_{j}\right)\right. \\
& \left.+K\left(x_{s}, x_{s}, c_{s}\right)\right)=g\left(x_{s}\right), \quad s=2,3,4, \ldots, n .
\end{aligned}
$$

Proposition 4. Given equation (1) and that $f(x), g(x) \in C[a, b], f^{\prime}(x)$ and $g^{\prime}(x)$ exist and are bounded in $[a, b], K(x, y, u) \in C[a, b] \times[a, b] \times[a, b]$ and $(\partial / \partial x)(K(x, y, u))$ and $(\partial / \partial y)(K(x, y, u))$ exist and are bounded in $[a, b] \times[a, b] \times[a, b]$. Furthermore, $K(x, y, u)$ satisfies the following condition:

$$
\begin{aligned}
& \left|\int_{a}^{b}(K(x, t, f(t))-K(x, t, u(t))) \mathrm{d} t\right| \\
& <L M \max _{x \in[a, b]}|f(x)-u(x)|,
\end{aligned}
$$

where $|L M|<1$. Let $n$ be an integer, $h=(b-a) / n$; let $x_{i}=a+i h, f_{i}=f\left(x_{i}\right), i=0,1,2, \ldots, n . c_{0}, c_{1}, \ldots, c_{n}$ satisfies the nonlinear system (17):

$$
f^{*}(x)=\sum_{k=0}^{n} c_{i} B_{1, h}(x-k h) .
$$


Then,

$$
\left\|f^{*}(x)-f(x)\right\|_{[a, b]}=O\left(h^{2}\right)
$$

where $f(x)$ is the exact solution of equation (1).

4.2. Method 2-V for Solving the Volterra Integral Equation. For more accurate solution, we apply $L_{3}(x / h)$. Again, we let $h=(b-a) / n$ and $x_{i}=a+i h, i=-1,0, \ldots, n+1$

Furthermore, let $L_{3, h}(x)=L_{3}(x / h)$ be the cardinal spline given in Section 3, and

$$
\begin{aligned}
f(x) & =\sum_{k=-1}^{n+1} c_{k} L_{3, h}\left(x-x_{k}\right), K(x, t, y(t)), \\
K(x, t, y(t)) & =\sum_{i=-1}^{n+1} \sum_{j=-1}^{n+1} K\left(x_{i}, x_{j}, c_{j}\right) L_{3, h}\left(x-x_{i}\right) L_{3, h}\left(t-x_{j}\right), \\
g(x) & =\sum_{k=-1}^{n+1} g\left(x_{k}\right) L_{3, h}\left(x-x_{k}\right) .
\end{aligned}
$$

Since the support of $L_{3, h}\left(x-x_{-1}\right)$ and $L_{3, h}\left(x-x_{n+1}\right)$ is wider than $[a, b]$, we need values that define

$$
\begin{aligned}
K\left(x_{-1}, x_{j}\right)= & 3 K\left(x_{0}, x_{j}\right)-3 K\left(x_{1}, x_{j}\right)+K\left(x_{2}, x_{j}\right), \quad j=0,1, \ldots, n, \\
K\left(x_{i}, x_{-1}\right)= & 3 K\left(x_{i}, x_{0}\right)-3 K\left(x_{i}, x_{1}\right)+K\left(x_{i}, x_{2}\right), \quad i=0,1, \ldots, n, \\
K\left(x_{-1}, x_{-1}\right)= & 3 K\left(x_{0}, x_{0}\right)-3 K\left(x_{1}, x_{0}\right)+K\left(x_{2}, x_{0}\right)+3 K\left(x_{0}, x_{1}\right)-3 K\left(x_{1}, x_{1}\right) \\
& +K\left(x_{2}, x_{1}\right)-\left(3 K\left(x_{0}, x_{2}\right)-3 K\left(x_{1}, x_{2}\right)+K\left(x_{2}, x_{2}\right)\right), \\
g\left(x_{-1}\right)= & 3 g\left(x_{0}\right)-3 g\left(x_{1}\right)+g\left(x_{2}\right), \\
K\left(x_{n+1}, x_{j}\right)= & 3 K\left(x_{n}, x_{j}\right)-3 K\left(x_{n-1}, x_{j}\right)+K\left(x_{n-2}, x_{j}\right), \quad j=0,1, \ldots, n, \\
K\left(x_{i}, x_{n+1}\right)= & 3 K\left(x_{i}, x_{n}\right)-3 K\left(x_{i}, x_{n-1}\right)+K\left(x_{i}, x_{2}\right), \quad i=0,1, \ldots, n, \\
K\left(x_{n+1}, x_{n+1}\right)= & 3 K\left(x_{n}, x_{n}\right)-3 K\left(x_{n-1}, x_{n}\right)+K\left(x_{n-2}, x_{n}\right)+3 K\left(x_{n}, x_{n-1}\right)-3 K\left(x_{n-1}, x_{n-1}\right) \\
& +K\left(x_{n-2}, x_{n-1}\right)-\left(3 K\left(x_{n}, x_{n-2}\right)-3 K\left(x_{n-1}, x_{n-2}\right)+K\left(x_{n-2}, x_{n-2}\right)\right), \\
g\left(x_{n+1}\right)= & 3 g\left(x_{n}\right)-3 g\left(x_{n-1}\right)+g\left(x_{n-2}\right) .
\end{aligned}
$$

Let $\left\{c_{0}, c_{1}, \ldots, c_{n}\right\}$ be the unknown coefficients to be determined and $c_{-1}=3 c_{0}-3 c_{1}+c_{2}$ and $c_{n+1}=3 c_{n}-3 c_{n-1}+c_{n-2}$. Plug into the integral equation (1); then, we have

$$
\begin{aligned}
& \sum_{k=-1}^{n+1} c_{k} L_{3, h}\left(x-x_{k}\right)-\lambda \int_{a}^{x} \sum_{i=-1}^{n+1} \sum_{j=-1}^{n+1} K\left(x_{i}, x_{j}, c_{j}\right) L_{3, h} \\
& \quad\left(x-x_{i}\right) L_{3, h}\left(t-x_{j}\right) \mathrm{d} t=\sum_{k=-1}^{n+1} g\left(x_{k}\right) L_{3, h}\left(x-x_{k}\right), \quad x \in[a, b] .
\end{aligned}
$$

Let $x=x_{i}$; we arrive at

$$
\begin{aligned}
& c_{s}-\sum_{j=-1}^{n+1} K\left(x_{s}, x_{j}, c_{j}\right) \int_{a}^{x_{s}} L_{3, h}\left(t-x_{j}\right) \mathrm{d} t \\
& =g\left(x_{s}\right), \quad s=0,1,2,3,4, \ldots, n,
\end{aligned}
$$

which is still a relatively simple system of equations. For the convergence rate of solution of Volterra integral equation (1), we have a similar result.

Proposition 5. Given that $f(x), g(x) \in C^{3}[a, b], f^{(4)}(x)$ and $g^{(4)}(x)$ exist and are bounded in $[a, b]$,
$K(x, t, y) \in C^{3}([a, b] \times[a, b] \times[c, d])$.

Furthermore, $K(x, t, y)$ satisfies the following condition:

$$
\left|\int_{a}^{b}(K(x, t, f(t))-K(x, t, u(t))) \mathrm{d} t\right|<L M \max _{x \in[a, b]}|f(x)-u(x)| .
$$

where $|L M|<1$. Let $n$ be an integer, $h=(b-a) / n$; let $x_{i}=a+i h, f_{i}=f\left(x_{i}\right), i=0,1,2, \ldots, n . \quad c_{0}, c_{1}, \ldots, c_{n}$ satisfies system (35):

$$
f^{*}(x)=\sum_{k=-1}^{n+1} c_{k} L_{3, h}\left(x-x_{k}\right) .
$$

Then,

$$
\begin{aligned}
\left\|f^{*}(x)-f(x)\right\|_{[a, b \%]} & =O\left(h^{3}\right), \\
f^{*}(x)-f(x)_{[a, b]} & =O\left(h^{3}\right),
\end{aligned}
$$

where $f(x)$ is the exact solution of equation (1).

\section{Numerical Examples}

Example 1 (from [1]). Solve 


$$
u(x)=f(x)+\int_{0}^{x} \frac{e^{x} u^{2}(t)}{1+e^{x+t}} \mathrm{~d} t,
$$

where $f(x)=e^{x / 2}-\ln \left(\left(1+e^{2 x}\right) /\left(1+e^{x}\right)\right)$. For comparison, we found that the exact solution is $u(x)=e^{x / 2}$.

To find the numerical solution in $[0,1]$, we let $h=0.1, n=10, x_{i}=i h, i=0,1,2, \ldots, 10, r(x, t)=\left(e^{x} / 1+\right.$

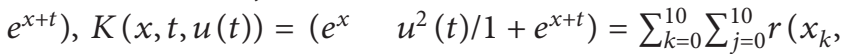
$\left.t_{j}\right) c_{j}^{2} B_{1, h} \%\left(x-x_{k}\right) \cdot B_{1, h}\left(t-x_{j}\right)$, and $f(x)=\sum_{k=0}^{10} f\left(x_{k}\right) B_{1, h}$ $\left(x-x_{k}\right), u(x)=\sum_{k=0}^{10} c_{k} B_{1, h}\left(x-x_{k}\right)$. Plug into integral equation (29), and let $x=x_{i}$; we obtain the following nonlinear system:

$$
\begin{array}{r}
c_{i}=f\left(x_{i}\right)+\int_{0}^{x_{i}} \sum_{j=0}^{10} r\left(x_{i}, t_{j}\right) c_{j}^{2} \cdot B_{1, h}\left(t-x_{j}\right) \mathrm{d} t \\
i=0,1,2, \ldots, 10 .
\end{array}
$$

Since it is a triangular system, which means, the first equation only contains $c_{0}$ and the $i$ th equation only contains $c_{0}$, $c_{1}, \ldots, c_{n-1}, i=0,1,2, \ldots, 10$, it is relatively easy to solve; the solution is not unique because the quadratic nature $c_{0}$ is unique, so we choose solution $c_{1}$ that is close to $c_{0}$, and so on. We arrive at $\left[c_{0}, c_{1}, c_{2}, c_{3}, c_{4}, c_{5}, c_{6}, c_{7}, c_{8}, c_{9}, c_{10}\right]=[1,1.05127,1.10516$, $1.16182,1.221378,1.28399,1.34980,1.41899,1.49173$, $1.568120,1.64859]$, with error: $\left|c_{i}-e^{x_{i} / 2}\right|<1.5 \times 10^{-4}$.

Let $\quad h=(1 / 20), n=20, x_{i}=i h, \quad i=0,1,2, \ldots, 20$, $r(x, t)=\left(e^{x} / 1+e^{x+t}\right), \quad K(x, t, u(t))=\left(e^{x} u^{2}(t) / 1+e^{x+t}\right)=$ $\sum_{k=0}^{20} \sum_{j=0}^{20} r\left(x_{k}, t_{j}\right) c_{j}^{2} B_{1, h}\left(x-x_{k}\right) \cdot B_{1, h}\left(t-x_{j}\right), f(x)=\sum_{k=0}^{20}$ $f\left(x_{k}\right) B_{1, h}\left(x-x_{k}\right)$, and $u(x)=\sum_{k=0}^{20} c_{k} B_{1, h}\left(x-x_{k}\right) ;$ plug into integral equation (29) and let $x=x_{i}$; we obtain the following nonlinear system:

$$
\begin{array}{r}
c_{i}=f\left(x_{i}\right)+\int_{0}^{x_{i}} \sum_{j=0}^{20} r\left(x_{i}, t_{j}\right) c_{j}^{2} \cdot B_{1, h}\left(t-x_{j}\right) \mathrm{d} t \\
i=0,1,2, \ldots, 20 .
\end{array}
$$

Since it is still a triangular system, it is relatively easy to solve; the solution is not unique because the quadratic nature $c_{0}$ is unique, so we choose solution $c_{1}$ that is close to $c_{0}$, and so on. We arrive at $\left[c_{0}, c_{1}, c_{2}, c_{3}, c_{4}, c_{5}, c_{6}, c_{7}, c_{8}, c_{9}\right.$, $\left.c_{10}, c_{11}, c_{12}, c_{13}, c_{14}, c_{15}, c_{16}, c_{17}, c_{18}, c_{19}, c_{20}\right]=[1, \quad 1.025315$, $1.051271,1.077883,1.105169,1.133146,1.161831,1.191241$, $1.221396,1.252315,1.284015,1.316519,1.349845,1.384014$, $1.419049,1.454971,1.491801,1.529565,1.568284,1.607983$, 1.648688 ] with error $<3.6 \times 10^{-5}$.

Example 2 (from [1]). Solve

$$
u(x)=f(x)-\int_{0}^{x}\left(u^{2}(t)+u(t)\right) \mathrm{d} t,
$$

where $f(x)=(3 / 2)-(1 / 2) e^{-2 x}$ and the exact solution is $u(x)=e^{-x}$.

To find the numerical solution in $[0,1]$, we let $h=0.1, n=10, x_{i}=i h, i=0,1,2, \ldots, 10, \quad r(x, t)=1$, $K(x, t, u(t))=\sum_{k=0}^{10} \sum_{j=0}^{10} r\left(x_{k}, t_{j}\right)\left(c_{j}^{2}+c_{j}\right) B_{1, h}\left(x-x_{k}\right) \cdot B_{1, h}$ $\left(t-x_{j}\right), \quad f(x)=\sum_{k=0}^{10} f\left(x_{k}\right) B_{1, h}\left(x-x_{k}\right)$, and $u(x)=\sum_{k=0}^{n+1}$ $c_{k} B_{1, h}\left(x-x_{k}\right)$; plug into integral equation (32), and let $x=x_{i}$; we obtain the following nonlinear system:

$$
\begin{aligned}
c_{i}= & f\left(x_{i}\right)-\int_{0}^{x_{i}} \sum_{j=0}^{10} r\left(x_{i}, t_{j}\right)\left(c_{j}^{2}+c_{j}\right) \\
& \cdot B_{1, h}\left(t-x_{j}\right) \mathrm{d} t, \quad i=0,1,2, \ldots, 10 .
\end{aligned}
$$

It is a triangular system again, so it is relatively easy to solve. We arrive at $\left[c_{0}, c_{1}, c_{2}, c_{3}, c_{4}, c_{5}, c_{6}, c_{7}, c_{8}, c_{9}, c_{10}\right]=$ $[1,0.904602, \quad 0.818183,0.740144, \quad 0.669608,0.605795$, $0.548075,0.495864,0.448631,0.405902,0.367245]$, with the error: $\left|c_{i}-u\left(x_{i}\right)\right|<8 \times 10^{-4}$ (in the paper [1], the error was $10^{-3}$ for much smaller $h$ ).

Let $h=0.05, n=20, x_{i}=i h, i=0,1,2, \ldots, 20, r(x, t)=$ $(\sin (x-t)+1), \quad f(x)=\cos x+(1 / 2) \sin x+(1 / 2) x \cos x$, $K(x, t, u(t))=\sum_{k=} 0^{20} \sum_{j=0}^{10} r\left(x_{k}, t_{j}\right)\left(c_{j}\right) B_{1, h}\left(x-x_{k}\right) \cdot B_{1, h}$ $\left(t-x_{j}\right), \quad f(x)=\sum_{k=0}^{20} f\left(x_{k}\right) B_{1, h}\left(x-x_{k}\right), \quad$ and $u(x)=$ $\sum_{k=0}^{20} c_{k} B_{1, h}\left(x-x_{k}\right)$; plug into integral equation (33) and let $x=x_{i}$; we obtain the following nonlinear system:

$$
\begin{array}{r}
c_{i}=f\left(x_{i}\right)-\int_{0}^{x_{i}} \sum_{j=0}^{20} r\left(x_{i}, t_{j}\right)\left(c_{j}\right) \cdot B_{1, h}\left(t-x_{j}\right) \mathrm{d} t \\
i=0,1,2, \ldots, 10 .
\end{array}
$$

We arrive at $\left[c_{0}, c_{1}, c_{2}, c_{3}, c_{4}, c_{5}, c_{6}, c_{7}, c_{8}, c_{9}, c_{10}, c_{11}\right.$, $\left.c_{12}, c_{13}, c_{14}, c_{15}, c_{16}, c_{17}, c_{18}, c_{19}, c_{20}\right]=[1,0.951144,0.904745$, $0.860597,0.818598,0.778651,0.740656,0.704517,0.670143$, $0.637447,0.606348,0.576766,0.548628,0.521864,0.496405$, $0.472189,0.449155,0.427245,0.406403,0.386579,0.367721]$ with error $<2 \times 10^{-4}$.

Example 3 (from [3]). Solve $\int_{0}^{x}(\sin (x-t)+1) \cos (y(t)) \mathrm{d} t$ $=(1 / 2) x \sin x+\sin x, 0 \leq x \leq 1$.

By solving $u(x)=\cos (y(x))$ first, it becomes a linear equation. To change it into Volterra integral equation of the second kind, we differentiate the equation and obtain the following:

$$
u(x)+\int_{0}^{x} \cos (x-t) u(t) \mathrm{d} t=\cos x+\frac{1}{2} \sin x+\frac{1}{2} x \cos x
$$

To find the numerical solution in $[0,1]$, we let $h=0.05$, $n=20, x_{i}=i h, i=0,1,2, \ldots, 20, \quad r(x, t)=\cos (x-t)$, $f(x)=\cos x+(1 / 2) \sin x+(1 / 2) x \cos x, \quad K(x, t, u(t))=$ $\sum_{k=0}^{20} \sum_{j=0}^{20} r\left(x_{k}, t_{j}\right)\left(c_{j}\right) B_{1, h}\left(x-x_{k}\right) \cdot B_{1, h}\left(t-x_{j}\right), f(x)=\sum_{k=0}^{20}$ $f\left(x_{k}\right) B_{1, h}\left(x-x_{k}\right)$, and $u(x)=\sum_{k=0}^{20} c_{k} B_{1, h}\left(x-x_{k}\right)$; plug into integral equation (35), and let $x=x_{i}$; we obtain the following nonlinear system:

$$
\begin{array}{r}
c_{i}=f\left(x_{i}\right)-\int_{0}^{x_{i}} \sum_{j=0}^{20} r\left(x_{i}, t_{j}\right)\left(c_{j}\right) \cdot B_{1, h}\left(t-x_{j}\right) \mathrm{d} t \\
i=0,1,2, \ldots, 10 .
\end{array}
$$

We arrive at $\left[c_{0}, c_{1}, c_{2}, c_{3}, c_{4}, c_{5}, c_{6}, c_{7}, c_{8}, c_{9}, c_{10}, c_{11}\right.$, $\left.c_{12}, c_{13}, c_{14}, c_{15}, c_{16}, c_{17}, c_{18}, c_{19}, c_{20}\right]=[1,0.999166,0.994962$, 0.989190, 0.979979, 0.969336, 0.955202, 0.939805, 0.920877, 
$0.900891,0.877347,0.852984,0.825046,0.796563,0.764495$, $0.732193,0.696299,0.660518,0.621139,0.582251,0.539763]$. The error $\left|c_{i}-u\left(x_{i}\right)\right|<5.4 \times 10^{-4}$.

Applying $\mathrm{L}_{3}$, we still let $h=0.1, n=10, x_{i}=i h, i=0$, $1,2, \ldots, 10, r(x, t)=(\sin (x-t)+1), f(x)=\cos x+(1 / 2)$ $\sin x+(1 / 2) x \cos x, K(x, t, u(t))=\sum_{k=-1}^{11} \sum_{j=-1}^{11} r\left(x_{k}, t_{j}\right)\left(c_{j}\right)$ $L_{3, h}\left(x-x_{k}\right) \cdot L_{3, h}\left(t-x_{j}\right), \quad f(x)=\sum_{k=-1}^{11} f\left(x_{k}\right) L_{3, h}\left(x-x_{k}\right)$, and $u(x)=\sum_{k=-1}^{11} c_{k} L_{3, h}\left(x-x_{k}\right)$; plug into integral equation (35), and let $x=x_{i}$, we obtain the following nonlinear system:

$$
\begin{aligned}
& \sum_{k=-1}^{11} c_{k} L_{3, h}\left(x-x_{k}\right)-\int_{a}^{x} \sum_{k=-1}^{11} \sum_{j=-1}^{11} r\left(x_{k}, t_{j}\right) c_{j} \\
& \cdot L_{3, h}\left(x-x_{k}\right) L_{3, h}\left(t-x_{j}\right) \mathrm{d} t=\sum_{k=-1}^{n+1} g\left(x_{k}\right) L_{3, h}\left(x-x_{k}\right), \\
& x \in[a, b],
\end{aligned}
$$

where $c_{-1}=3 c_{0}-3 c_{1}+c_{2}$ and $c_{n+1}=3 c_{n}-3 c_{n-1}+c_{n-2}$; we achieve $\left[c_{0}, c_{1}, c_{2}, c_{3}, c_{4}, c_{5}, c_{6}, c_{7}, c_{8}, c_{9}, c_{10}\right]=[1,0.99501$, $0.98007,0.95534,0.92107,0.87759,0.82534,0.76485,0.69$ $671,0.62162,0.54031]$ with error $\left|c_{i}-u\left(x_{i}\right)\right|<1.2 \times 10^{-5}$.

We use the same process, and let $h=0.05$ and $x_{i}=i h, i=0,1,2, \ldots, 20$, the result is $\left[c_{0}, c_{1}, c_{2}, c_{3}, c_{4}, c_{5}, c_{6}\right.$, $\left.c_{7}, c_{8}, c_{9}, c_{10}, c_{11}, c_{12}, c_{13}, c_{14}, c_{15}, c_{16}, c_{17}, c_{18}, c_{19}, c_{20}\right]=[1.0$, $0.99875,0.995,0.98877,0.98007,0.96891,0.95534,0.93937$, 0.92106, 0.90045, 0.87758, 0.85252, 0.82534, 0.79608, 0.76484, $0.73169,0.69671,0.65998,0.62161,0.58168,0.5403]$ with error: $<4.6 \times 10^{-4}$.

Example 4 (from [3]). $\quad \int_{0}^{x} e^{x-t} \ln (y(t)) \mathrm{d} t=e^{x}-x-1$, $0 \leq x \leq 1 .\left(y(x)=e^{x}\right)$

We change it to the linear Volterra integral equation of second kind:

$$
w(x)+\int_{0}^{x} e^{x-t} w(t) \mathrm{d} t=e^{x}-1, \quad 0 \leq x \leq 1,
$$

where $w(t)=\ln (y(t))$.

Let $h=0.1, n=10, x_{i}=i h, i=0,1,2, \ldots, 10, r(x, t)=$ $e^{x-t}, f(x)=e^{x}-1, K(x, t, u(t))=\sum_{k=0}^{10} \sum_{j=0}^{10} r\left(x_{k}, t_{j}\right)\left(c_{j}\right)$ $B_{1, h}\left(x-x_{k}\right) \cdot B_{1, h}\left(t-x_{j}\right), \quad f(x)=\sum_{k=0}^{10} f\left(x_{k}\right) B_{1, h}\left(x-x_{k}\right)$, and $u(x)=\sum_{k=0}^{n+1} c_{k} B_{1, h}\left(x-x_{k}\right)$; plug into integral equation (38), and let $x=x_{i}$; we obtain the following nonlinear system:

$$
\begin{array}{r}
c_{i}=f\left(x_{i}\right)-\int_{0}^{x_{i}} \sum_{j=0}^{10} r\left(x_{i}, t_{j}\right)\left(c_{j}\right) \cdot B_{1, h}\left(t-x_{j}\right) \mathrm{d} t \\
i=0,1,2, \ldots, 10 .
\end{array}
$$

We arrive at $\left[c_{0}, c_{1}, c_{2}, c_{3}, c_{4}, c_{5}, c_{6}, c_{7}, c_{8}, c_{9}, c_{10}\right]=$ $[0,0.100163,0 . \quad 200317,0.300463,0.400601,0.500730,0.60$ $0851,0.700964,0.801068,0.901164,1.00125]$. The error $\mid c_{i}-$ $u\left(x_{i}\right) \mid<4 \times 10^{-3}$.
Using the same process, let $h=0.05, n=20, x_{i}=i h, i=$ $0,1,2, \ldots, 20, \quad r(x, t)=e^{x-t}, \quad f(x)=e^{x}-x-1, \quad K(x, t$, $u(t))=\sum_{k=0}^{20} \sum_{j=0}^{20} r\left(x_{k}, t_{j}\right)\left(c_{j}\right) B_{1, h}\left(x-x_{k}\right) \cdot B_{1, h}\left(t-x_{j}\right)$, $f(x)=\sum_{k=0}^{20} f\left(x_{k}\right) B_{1, h}\left(x-x_{k}\right)$, and $u(x)=\sum_{k=0}^{n+1} c_{k} B_{1, h}(x-$ $x_{k}$ ); plug into integral equation (38), and let $x=x_{i}$; we obtain the following nonlinear system:

$$
\begin{array}{r}
c_{i}=f\left(x_{i}\right)-\int_{0}^{x_{i}} \sum_{j=0}^{20} r\left(x_{i}, t_{j}\right)\left(c_{j}\right) \cdot B_{1, h}\left(t-x_{j}\right) \mathrm{d} t, \\
\mathrm{i}=0,1,2, \ldots, 20 .
\end{array}
$$

We arrive at $\left[\mathrm{c}_{01}, \mathrm{c}_{2}, \mathrm{c}_{3}, \mathrm{c}_{4}, \mathrm{c}_{5}, \mathrm{c}_{6}, \mathrm{c}_{7}, \mathrm{c}_{8}, \mathrm{c}_{9}, \mathrm{c}_{10}, \mathrm{c}_{11}\right.$, $\left.\mathrm{c}_{12}, \mathrm{c}_{13}, \mathrm{c}_{14}, \mathrm{c}_{15}, \mathrm{c}_{16}, \mathrm{c}_{17}, \mathrm{c}_{18}, \mathrm{c}_{19}, \mathrm{c}_{20}\right]=[0,0.0500206,0.100041$, $0.150060,0.200079,0.250098,0.300116,0.350133,0.400150,0$. $450166,0.50018,0.550198,0.600213,0.650227,0.700241,0.75$ 0254, 0.800267, 0.850279, 0.900291, 0.950302, 1.00031]. The error: $\left|c_{i}-u\left(x_{i}\right)\right|<8.5 \times 10^{-4}$.

Applying $L_{3}$, we still let $h=0.05, n=20, x_{i}=i h, i=$ $0,1,2, \ldots, 20, \quad r(x, t)=\sin (x-t)+1 f(x)=\cos x+(1 / 2)$ $\sin x+(1 / 2) x \cos x, K(x, t, u(t))=\sum_{k=-1}^{21} \sum_{j=-1}^{21} r\left(x_{k}, t_{j}\right)\left(c_{j}\right)$ $L_{3, h}\left(x-x_{k}\right) \cdot L_{3, h}\left(t-x_{j}\right), f(x)=\sum_{k=-1}^{21} f\left(x_{k}\right) L_{3, h}\left(x-x_{k}\right)$, and $u(x)=\sum_{k=-1}^{21} c_{k} L_{3, h}\left(x-x_{k}\right)$; plug into integral equation (38), and let $x=x_{i}$; we obtain the following nonlinear system:

$$
\begin{aligned}
& \sum_{k=-1}^{21} c_{k} L_{3, h}\left(x-x_{k}\right)-\int_{a}^{x} \sum_{k=-1}^{21} \sum_{j=-1}^{21} r\left(x_{k}, t_{j}\right) c_{j} \\
& \cdot L_{3, h}\left(x-x_{k}\right) L_{3, h}\left(t-x_{j}\right) \mathrm{d} t=\sum_{k=-1}^{21} g\left(x_{k}\right) L_{3, h}\left(x-x_{k}\right), \\
& x \in[a, b],
\end{aligned}
$$

where $c_{-1}=3 c_{0}-3 c_{1}+c_{2}$ and $c_{n+1}=3 c_{n}-3 c_{n-1}+c_{n-2}$; we achieve $\left[\mathrm{c}_{0}, \mathrm{c}_{1}, \mathrm{c}_{2}, \mathrm{c}_{3}, \mathrm{c}_{4}, \mathrm{c}_{5}, \mathrm{c}_{6}, \mathrm{c}_{7}, \mathrm{c}_{8}, \mathrm{c}_{9}, \mathrm{c}_{10}, \mathrm{c}_{11}, \mathrm{c}_{12}, \mathrm{c}_{13}, \mathrm{c}_{14}, c\right.$ $\left.c_{15}, c_{16}, c_{17}, c_{18}, c_{19}, c_{20}\right]=[0.0, \quad 0.04,0.999998,0.09999996$, $0.1499999,0.1999999,0.2499999,0.2999999,0.3499999,0.39$ 999986, 0.44999984, 0.49999982, 0.54999980, 0.59999979, $0.64999977,0.69999976,0.74999974,0.79999972,0.8499997$ $1,0.89999969,0.94999968,0.99999967]$. The error $\left|c_{i}-u\left(x_{i}\right)\right|<3.5 \times 10^{-7}$ (in paper [1], the error was $10^{-4}$ for a much smaller $h=(1 / 32)$, they achieve the error $4.7 \times 10^{-7}$ with $h=(1 / 512))$.

\section{Conclusion}

The proposed method is a simple and effective procedure for solving nonlinear Volterra integral equations of the second kind. The methods can be adapted easily to the Volterra integral equations of the first kind. The methods can also be extended to the Fredholm and Volterra integral equations of the first kind or the second kind, where the integral is on an infinite set. When the higher degree cardinal splines are applied to nonlinear integral equations, the resulting system of coefficients will be a little more complicated nonlinear 
system, which takes more time and effort to solve. Compared with the recent papers [1] and [3], our method is more effective and efficient. Furthermore, we could use higher dimensional spline functions to solve higher dimensional integral equations.

\section{Data Availability}

The data used to support the findings of this study are available from the corresponding author upon request.

\section{Conflicts of Interest}

The authors declare that there are no conflicts of interest regarding the publication of this paper.

\section{Acknowledgments}

The work was funded by the Fostering Master's Degree Empowerment Point Project of Hefei University under Grant no. 2018xs03 and the Project Foundation of Scientific Research, Education Department of Anhui Province, under Grant no. KJ2019A0846. This research was partially supported by the research funds of the University of La Verne. Ms. Jiahuan Huang was an undergraduate student at the University of La Verne, who did some numerical calculations. The authors thank the organizers of the 8th International Conference on Computational Methods for accepting this paper for presentation.

\section{References}

[1] I. Aziz and Siraj-ul-Islam, "New algorithms for the numerical solution of nonlinear Fredholm and Volterra integral equations using Haar wavelets," Journal of Computational and Applied Mathematics, vol. 239, pp. 333-345, 2013.

[2] A. D. Polyanin, Handbook of Integral Equations, CRC Press LLC, Boca Raton, FL, USA, 1998.

[3] I. Singh and S. Kumar, "Haar wavelet method for some nonlinear Volterra integral equations of the first kind," Journal of Computational and Applied Mathematics, vol. 292, pp. 541-552, 2016.

[4] X. Liu and J. Xie, "Numerical methods for solving systems of Fredholm integral equations with cardinal splines," AIP Conference Proceedings, vol. 1637, p. 590, 2014.

[5] X. Liu, Z. Liu, and J. Xie, "Solving systems of Volterra integral equations with cardinal splines," Journal of Applied Mathematics and Physics (JAMP), vol. 3, no. 11, pp. 1422-1430, 2015.

[6] J.-B. Liu, C. Wang, S. Wang, and B. Wei, "Zagreb indices and multiplicative zagreb indices of eulerian graphs," Bulletin of the Malaysian Mathematical Sciences Society, vol. 42, no. 1, pp. 67-78, 2019.

[7] I. J. Schoenberg, "Contributions to the problem of approximation of equidistant data by analytic functions," Quarterly of Applied Mathematics, vol. 4, pp. 45-99, 1946.

[8] C. K. Chui, Multivariate Splines, SIAM, Philadelphia, PA, USA, 1988.

[9] X. Liu, "Bivariate cardinal spline functions for digital signal processing," in Trends in Approximation Theory, K. Kopotum, T. Lyche, and M. Neamtu, Eds., pp. 261-271, Vanderbilt University, Nashville, TN, USA, 2001.
[10] X. Liu, "Univariate and bivariate orthornormal splines and cardinal splines on compact supports," Journal of Computational and Applied Mathematics, vol. 195, no. 1-2, pp. 93-105, 2006.

[11] X. Liu, "Interpolation by cardinal exponential splines," The Journal of Information and Computational Science, vol. 4, no. 1, pp. 179-194, 2007.

[12] X. Liu, "Interpolation by cardinal trigonometric splines," International Journal of Pure and Applied Mathematics, vol. 40, no. 1, pp. 115-122, 2007.

[13] I. Schoenberg, "On trigonometric spline functions," Indiana University Mathematics Journal, vol. 13, no. 5, pp. 795-825, 1964. 